\title{
Evolution of the Spallation Neutron Source Ring Lattice
}

\author{
J. Wei*, N. Catalan-Lasheras*, A. Fedotov*, C.J. Gardner*, Y.Y. Lee*, \\ Y. Papaphilippou* , D. Raparia* , N. Tsoupas* and J. Holmes ${ }^{\dagger}$ \\ ${ }^{*}$ Brookhaven National Laboratory, Upton, NY 11973, USA \\ † Oak Ridge National Laboratory, Oak Ridge, TN 37831, USA
}

\begin{abstract}
Requirements of minimum beam loss for hand-on maintenance and flexibility for future operations are essential for the lattice design of the Spallation Neutron Source (SNS) accumulator ring. During the past seven years, the lattice has evolved from an all-FODO to a FODO/doublet hybrid, the circumference has been increased to accommodate for a higher energy foreseen with a super-conducting RF linac, and the layout has evolved from an $\alpha$ - to an $\Omega$ - geometry. Extensive studies are performed to determine working points that accommodate injection painting and minimize beam losses due to space charge and resonances. In this paper, we review the evolution of the SNS ring lattice and discuss the rationales.
\end{abstract}

TABLE 1. Major lattice parameters of the SNS ring.

\begin{tabular}{lll}
\hline Quantity & Value & Unit \\
\hline Circumference & 248.0 & $\mathrm{~m}$ \\
Kinetic energy & 1 & $\mathrm{GeV}$ \\
Repetition rate & 60 & $\mathrm{~Hz}$ \\
Number of protons per pulse & 1.6 & $10^{14}$ \\
Ring dipole field & 0.792 & $\mathrm{~T}$ \\
Unnormalized full emittance, $\mathrm{H}+\mathrm{V}$ & 240 & $\pi \mu \mathrm{m}$ \\
Betatron acceptance & 480 & $\pi \mu \mathrm{m}$ \\
Momentum acceptance (full beam) & \pm 2 & $\%$ \\
Number of super-periods & 4 & \\
Nominal tunes $\gamma_{x}, v_{y}$ & $6.23,6.20$ & \\
Transition energy, $\gamma_{T}$ & 5.3 & \\
Maximum $\beta_{x}, \beta_{y}$ & $27.9,15.7$ & $\mathrm{~m}$ \\
Maximum $\beta_{x}, \beta_{y}$ in arc & $12.9,13.3$ & $\mathrm{~m}$ \\
Maximum ratio $\beta_{\max } / \beta_{\min }(\mathrm{H}, \mathrm{V})$ & $11.6,7.5$ & $\mathrm{~m}$ \\
Maximum dispersion $D_{x}$ & 4.0 & $\mathrm{~m}$ \\
Natural chromaticity $\left(\xi_{x}, \xi_{y}\right)$ & $-7.9,-6.9$ & \\
\hline
\end{tabular}

\section{INTRODUCTION}

The SNS project is designed to reach an average beam power above 1.4 MW for pulsed neutron production $[1,2]$. The accumulator ring, operating at a fixed energy of $1 \mathrm{GeV}$, compresses at $60 \mathrm{~Hz}$ repetition rate $1 \mathrm{~ms}$ beam pulses containing $1.6 \times 10^{14}$ protons to 650 ns bunches for delivery onto the target. Table 1 lists major lattice parameters of the ring.

\section{DESIGN EVOLUTION}

As shown in Fig. 1, the ring presently has a fourfold lattice symmetry containing four dispersion-free

\footnotetext{
${ }^{1}$ SNS is managed by UT-Battelle, LLC, under contract DE-AC0500OR22725 for the U.S. Department of Energy. SNS is a partnership of six national laboratories: Argonne, Brookhaven, Jefferson, Lawrence Berkeley, Los Alamos, and Oak Ridge.
}

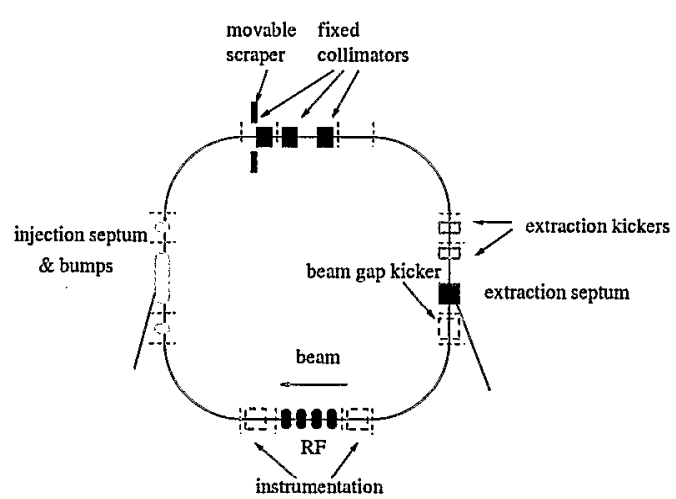

FIGURE 1. Functions of the SNS accumulator ring.

straight sections, each housing injection, collimation, radio-frequency $(\mathrm{RF})$ system, and extraction [3].

\section{Layout}

At pre-construction stage, the lattice's four-fold symmetry [4] was chosen against a three-fold symmetry [5] for its separate, dedicated function of each straight section, reduced maximum dispersion, and fewer structure resonances. Since the start of construction in 1999, several iterations have been made to the general layout of the ring. The early $\alpha$-geometry (Fig. 2) was later replaced by the $\Omega$-geometry to avoid the crossing of injection and extraction lines for easy tunnel access and maintenance. The circumference of the ring was also increased from $221 \mathrm{~m}$ to $248 \mathrm{~m}$ to accommodate the potential increase of injection energy up to $1.3 \mathrm{GeV}$ (longer injection-chicane magnets with lower field to avoid $\mathrm{H}^{-}$stripping), which is made possible by the adoption of superconducting RF linac, and to reduce bcam density and foil traversals. 


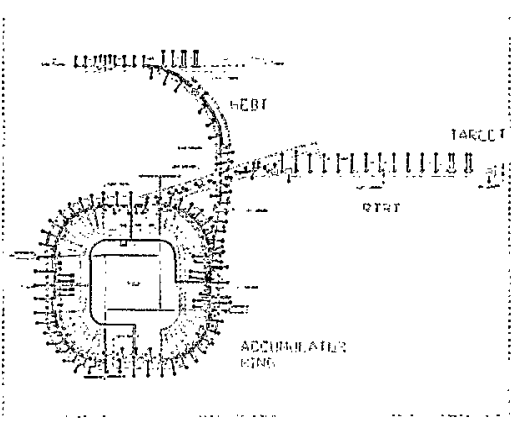

FIGURE 2. Original $\alpha$-layout of the SNS ring.

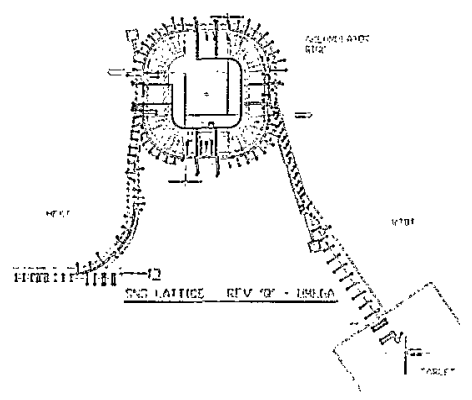

FIGURE 3. Present $\Omega$-layout of the SNS ring.

\section{Lattice}

Each achromatic arc consists of 4 FODO cells with $90^{\circ}$ horizontal phase advance [6]. In 1999, the original all-FODO structure (Fig. 4) was replaced a hybrid structure with matched FODO arcs and doublet straights (Fig. 5) [3]. The lattice combines the FODO's simplicity and ease of correction with the doublet's flexibility for injection and collimation. The $12.5 \mathrm{~m}$-long uninterrupted straight section with a flexible phase advance further improves collimation efficiency, and eliminates magnets between secondary collimators [7]. Comparing with the original lattice, matching between the arcs and the straights increases the arc acceplance by $50 \%$ with the same magnet aperture. The dipoles were also centered in each half cell to maximize the acceptance.

\section{Injection}

Injection at a dispersion-free region allows independently adjustable painting in the transverse (with orbit bumps in the ring) and longitudinal (with an energyspreading phase-modulated RF cavity in the HEBT) directions for a robust operation (Fig. 6) [8]. With the long straight section provided by the doublet lattice, the injection chicane is made independent from the lattice tuning

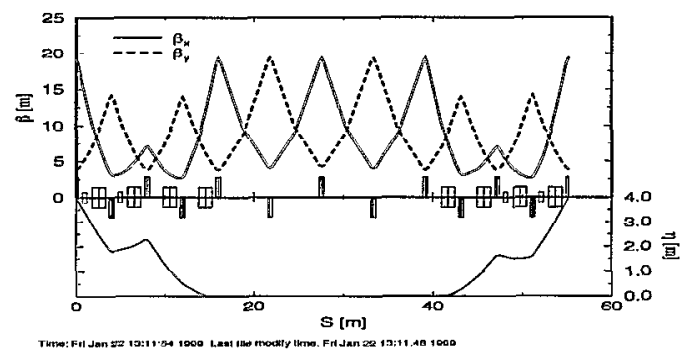

FIGURE 4. Original all-FODO lattice of the SNS ring.

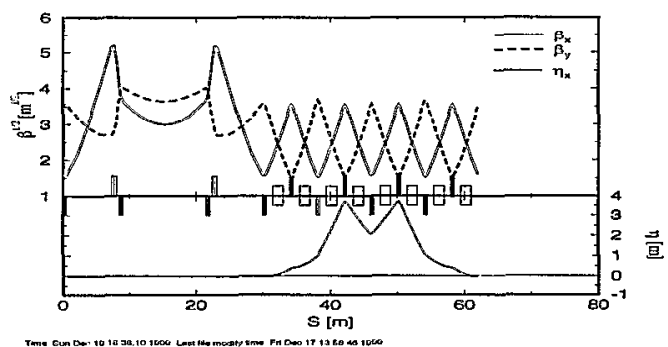

FIGURE 5. Present FODO-doublet lattice of the SNS ring.

(Fig. 7). The long straight section also accommodates possible future arrangement of laser-stripping injection $[9,10]$. A symmetric layout of the dynamic bump further simplifies the set-up of transverse painting [11].

\section{Tuning and Working Point}

Tume adjustment is provided by five families of quadrupole power supply. Horizontally, the phase advance across the arc is fixed to satisfy achromatic condition. The tuning range is limited to about one unit (from 6 to 7) (Fig. 8). Vertically, the tuning range is about two units (from 5 to 7 ) without significant perturbation from the injection bump [12].

The nominal working point $\left(v_{x}, v_{y}\right)$ is chosen to be $(6.23,6.2)$ near the coupling resonance $v_{x}=v_{y}$ (Fig. 9). With full coupling, a round beam is realized even with correlated painting [8]. The full tune spread is accommodated in the tune space without crossing lower-order structure resonances (Fig. 9). An alternative working $(6.4,6.3)$ is further away from the structure lines $2 v_{x}=$ 12 and $2 v_{y}=12$, although some third- and fourth-order

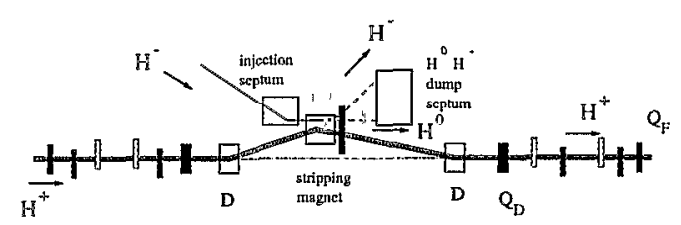

FIGURE 6. Original injection layout with a FODO straight. 


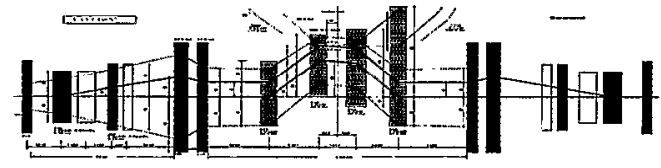

FIGURE 7. Present injection layout with a doublet straight.

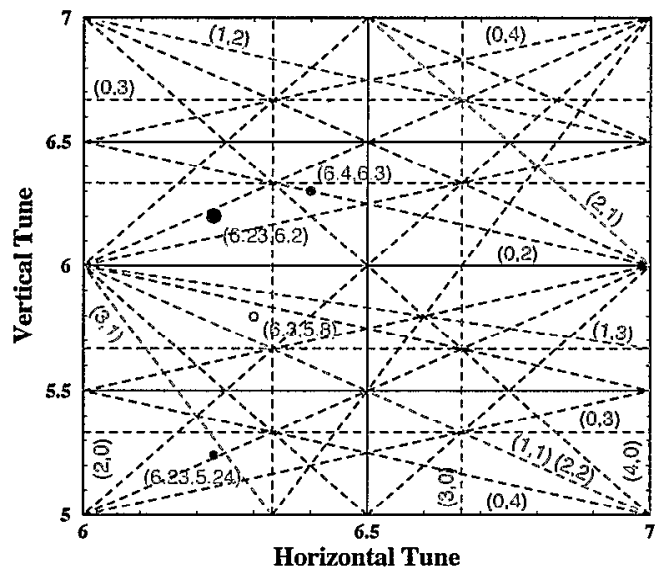

FIGURE 8. Nominal $(6.23,6.2)$ and alternative working points $(6.4,6.3),(6.23,5.24)$ in the transverse tune space. Sum structure resonances up to fourth order are shown in red. Other resonances up to third order are shown in black.

non-structure resonances must be crossed. A split-tune working point $(6.23,5.24)$ can also be used for decoupled operation [14]. Old working points $(5.82,5.8)$ and $(6.3,5.8)$ were abandoned due to matching difficulty and proximity to structure resonance, respectively.

\section{Perturbations and Correction}

The injection chicane produces a perturbation of about $5 \%$ in $\Delta \beta_{y} / \beta_{y}$, and $0.1 \mathrm{~m}$ in $D_{x}$ [12]. Together with the dynamic bump, the dispersions are perturbed by $0.25 \mathrm{~m}$ $(\mathrm{H})$ and $0.15 \mathrm{~m}(\mathrm{~V})$, respectively.

Correctors up to octupole order are used for orbit correction, decoupling, $\beta$-wave and vertical-dispersion correction, and resonance correction (Fig. 10) $[16,17]$.

\section{SUMMARY}

With four-fold symmetry, dispersion-free injection, collimation, RF, and extraction, and a matched FODOarc/doublet-straight optics, the SNS ring lattice is optimized for maximum acceptance, maximized dispersionfree space, flexible injection, high-efficiency collimation, and easy corrections.

We thank G. Rees and the ASAC committee for advice, and Y. Cho, J. Galambos, N. Malitsky, S. Tepikian, D. Trbojevic, W. Wan, and W.T. Weng for discussions.
SNS Working Point $\left(v_{x}, v_{y}\right)=(6.23,6.20)$

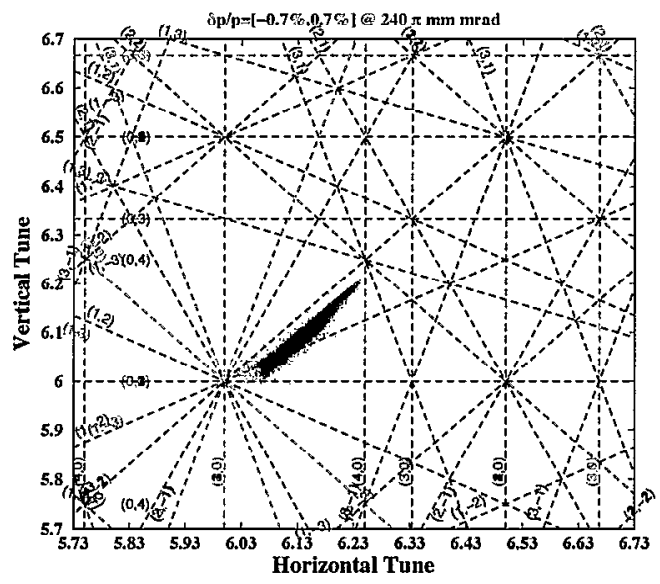

FIGURE 9. Tune spread of a beam containing $2 \times 10^{14}$ protons in the SNS ring. The computer-simulation results are obtained with the Unified-Accelerator-Libraries (UAL) package [13]. Structure resonances are indicated in red.

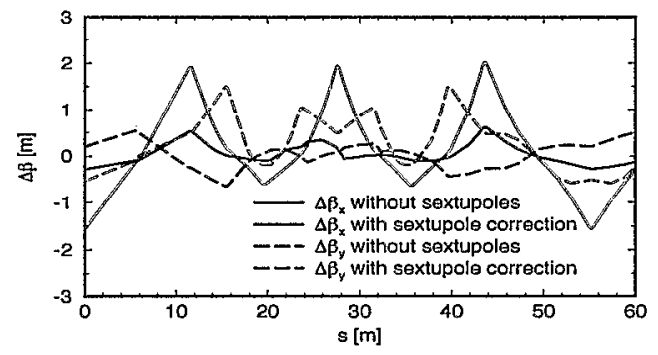

FIGURE 10. Correction of off-momentum $(\Delta p / p=-0.7 \%)$ optics using four-family sextupoles in a mismatched case [15].

\section{REFERENCES}

I. N. Holtkamp, EPAC (2002)

2. J. Wei, EPAC (2002)

3. J. Wei et al, Phys. Rev. ST-AB 3 (2000) 080101

4. Y.Y. Lee, BNL/NSNS/26 (1997)

5. A.G. Ruggiero, BNL/NSNS/1 (1997)

6. C.J. Gardner et al, PAC (1997) 962

7. N. Catalan-Lasheras et al, Phys. Rev. ST-AB 4 (2001) 010101; S. Cousineau et al, these proceedings

8. J. Wei et al, PAC (2001) 2560; EPAC (2000) 978

9. C.J. Gardner et al, PAC (1999) 3182

10. I. Yamane, Phys. Rev. ST-AB 1 (1998) 053501

11. J. Beebe-Wang et al, PAC (1999) 1743

12. J. Holmes et al, PAC (2001) 3618

13. N. Malitsky et al, PAC (1999) 2713

14. A.V. Fedotov et al., PAC (2001) 2848

15. N. Tsoupas et al, EPAC (2000) 1581

16. C.J. Gardner et al, BNL/SNS/31 (1997); BNL/SNS/37 (1997); BNL/SNS/40 (1997); BNL/SNS/82 (2000)

17. Y. Papaphilippou et al, PAC (2001) 1670 